\title{
EXACT TAIL ASYMPTOTICS OF A QUEUE WITH LRD INPUT TRAFFIC
}

\author{
M. Lelarge ${ }^{1}$ and A. Villani ${ }^{2}$
}

\begin{abstract}
In this work we compute the exact tail asymptotics of the stationary workload $W$, associated to a discretetime single server queue, with constant release rate, infinite buffer capacity, and with $M / G / \infty$ input traffic exhibiting long-range dependence. We choose a regularly varying distribution with parameter $\alpha>1$ for the general distribution $G$. We show that the exact asymptotics of the workload is a specific regularly varying function under some assumptions on the parameters.
\end{abstract}

\section{Introduction}

The rapid increase in the number and complexity of available communications services, such as the Web traffic [10] and the WAN traffic [15] for example, have made traditional traffic models based on exponential assumptions about inter-arrival times and resource holding requirements obsolete. The statistical profile of network traffic exhibits empirical correlations that decay to zero as a power rather than exponentially as those seen in traditional telephony.

For this reason, there has been broad interest in the literature in the study of service systems exhibiting some forms of long-range dependence of the input process in which the correlation functions are not summable.

Some models propose fractional Brownian motion and its discrete-time analog, namely fractional Gaussian noise, as input traffic processes with long-range dependence property. For instance, in $[9,11,13]$ the logarithm asymptotics of the workload for a single server queue is derived. All these studies arrive at the same result: the stationary workload associated to a single service queue is asymptotically Weibullian.

In this paper we take instead the busy server process of the $M / G / \infty$ queue as the input traffic process for a discrete-time single service system with constant release rate.

The fundamental observation about $M / G / \infty$ input traffic was made in [3]: if the distribution function $F$ of the service times in the $M / G / \infty$ queue has infinite variance, then the busy server process has nonsummable correlations, and thus provides an input process with long-range dependence for the queue of our interest.

In order to estimate the performances of the service system of interest, we will estimate the asymptotic tail, $\mathrm{P}(W>x)$, as $x \rightarrow \infty$, where $W$ is the stationary workload under the Loynes's stability conditions $[1,7,18]$. This study was made by many authors. In $[8,14]$ an upper and a lower bound for the logarithmic tail asymptotics was derived. A more precise result under reasonable general conditions was obtained for the Weibull, Lognormal and Pareto case. In the special case $F$ Pareto with tail parameter $\alpha>1$ we have

$$
\log \mathrm{P}(W>x) \sim-(\alpha-1) \log x
$$

where $a(x) \sim b(x)$ means $a(x) / b(x) \rightarrow 1, x \rightarrow \infty$. The main result of this work is an improvement of the previous result. Under the same assumptions on the parameters of the model but assuming only that $F$ is a distribution function with regularly varying tails with parameter $\alpha>1$, rather than simply Pareto, we get

$$
\mathrm{P}(W>x) \sim L(x) x^{-(\alpha-1)},
$$

where $L(x)$ is a specific slowly varying function.

The result (2) is in accordance with the exact asymptotics tail for a single server queue generated by the $M / G / \infty$ fluid input process [16], that is, the analogous queue in continuous time and under the same assumptions on the general distribution $G$.

The techniques used to obtain the result (2) are different from the large deviation arguments of $[8,14,16]$.

Our main tool is the analysis performed by Baccelli and Foss [2] of Veraverbeke's theorem [5, 17] on the asymptotic tail of the supremum of a random walk with i.i.d. subexponential increments with negative mean.

\footnotetext{
${ }^{1}$ ENS, Paris, France, e-mail: marc.lelarge@ens.fr

${ }^{2}$ Department of Information Engineering and Applied Mathematics, University of Salerno, via Ponte don Melillo, 84084 Fisciano (SA), Italy, e-mail: antonella.villani@tin.it
}

Proceedings of the XXVI International Seminar on Stability Problems for Stochastic Models, Sovata-Bai, Romania, August 27 - September 2, 2006. 
This analysis consists in identifying typical events responsible for the fact that the random walk has crossed the level $x$, up to higher-order probabilities as $x \rightarrow \infty$.

In order to use this analysis, we first derive an upper bound $W \leqslant U+V$, where $U$ and $V$ are independent and $V$ is the stationary workload of a single server queue with independent service times. This upper bound allows us to use Theorem 8 of [2], which shows that the probability $\mathrm{P}(W>x)$ is mainly due to one very big service time associated to a customer in the $M / G / \infty$ queue, whereas the others remain close to their mean.

\section{Model and main result}

We consider a discrete time single server queue with infinite buffer capacity and constant release rate of $a$ cells/slot with FIFO service discipline. The input process is represented by the sequence $\left\{\sigma_{k}, k=1,2, \ldots\right\}$, where $\sigma_{k+1}$ is the number of new cells arriving at the start of time slot $[k, k+1), k=1,2, \ldots$ Let $W_{k}$ denote the number of cells remaining in the buffer by the end of slot $[k-1, k)$ with $k \geqslant 1$, and suppose $Y$ is the number of cells in the buffer at time slot $[0,1)$, so that Lindley's recursion is

$$
\begin{cases}W_{0} & =Y \\ W_{k+1} & =\left[W_{k}+\sigma_{k+1}-a\right]^{+}, k=0,1, \ldots\end{cases}
$$

Let $W_{n}^{[Y]}$ be the solution of (3). If $\left\{\sigma_{k}, k=1,2, \ldots\right\}$ is a stationary and ergodic process, under the Loynes's stability assumption $[1,7,18] \mathrm{E}\left[\sigma_{1}\right]<a$, the system is stable in the sense that $W_{n}^{[Y]} \stackrel{\text { Law }}{\longrightarrow} W_{\infty}$, as $n \rightarrow \infty$ for some finite random variable $W_{\infty}$ and any initial condition $Y$.

\subsection{Stochastic Assumptions}

The sequence $\left\{\sigma_{k}, k=1,2, \ldots\right\}$ is generated by the $M / G / \infty$ input process of Cox [3] in the following way. Suppose that during time slot $[k-1, k), k=1,2, \ldots, b_{k}$ new customers arrive in the system. For $i=1, \ldots, b_{k}$, $X_{k, i}$ is the service time of the $i$ th customer. Customer $i$ is presented to its own server and begins service by the start of slot $[k, k+1), k \geqslant 1$. Moreover $b_{0}$ is the number of customers in the system at time 0 and for $i=1, \ldots, b_{0}$, $X_{0, i}$ is the residual service time of the $i$ th customer present in the queue at time 0 . Let $\sigma_{k}$ denote the number of busy servers, or equivalently, the number of customers still present in the system at the beginning of time slot $[k, k+1)$. The busy server process $\left\{\sigma_{k}, k=0,1,2, \ldots\right\}$ is what we refer to as the $M / G / \infty$ input process.

We have that

$$
\sigma_{k}=\sum_{i=1}^{b_{0}} 1_{\left\{X_{0, i}>k\right\}}+\sum_{j=1}^{k} \sum_{i=1}^{b_{j}} 1_{\left\{X_{j, i}>k-j\right\}}, \quad k \geqslant 0 .
$$

Now the random variables

$$
b_{0},\left\{b_{k}, k=1,2, \ldots\right\},\left\{X_{0, i}, i=1,2, \ldots, b_{0}\right\},\left\{X_{k, i}, k=1,2, \ldots, i=1,2, \ldots, b_{k}\right\},
$$

are assumed to satisfy the following assumptions:

(A1) all the sequences of random variables are mutually independent;

(A2) $\left\{b_{k}, k=1,2, \ldots\right\}$ are i.i.d. Poisson random variables with parameter $\lambda>0$ and $b_{0}$ is a Poisson random variable independent of everything else with parameter $\gamma>0$;

(A3) $\left\{X_{k, i}, k=1,2, \ldots, i=1,2, \ldots, b_{k}\right\}$ are i.i.d. random variables valued in $\mathbb{N}$ with the same distribution function of, say $X$, with $\mathrm{E}[X]<\infty$.

Moreover, $\left\{X_{0, i}, i=1,2, \ldots, b_{0}\right\}$ are assumed to be i.i.d random variables with the same distribution of the random variable $X_{0}$.

Moreover, we will assume that

(A4) $b_{0}$ has a Poisson distribution with mean $\gamma=\lambda \mathrm{E}[X]$;

(A5) $X_{0, i}$ has a distribution $\mathrm{P}\left(X_{0} \leqslant k\right)=\frac{1}{\mathrm{E}[X]} \sum_{n=1}^{k} \mathrm{P}(X \geqslant n)$, for $k=1,2, \ldots$. 
Proposition 1 ([3], [12]). Under the assumptions $(A 1),(A 2),(A 3),(A 4)$, and $(A 5)$ the process $\left\{\sigma_{k}, k=\right.$ $0,1,2, \ldots\}$ is stationary, ergodic, and reversible such that

$$
\gamma=\mathrm{E}\left[\sigma_{k}\right]=\lambda \mathrm{E}[X], \quad \forall k \geqslant 0
$$

Its covariance function is given by

$$
\Gamma(h)=\operatorname{cov}\left(\sigma_{k}, \sigma_{k+h}\right)=\lambda \mathrm{E}[X] \mathrm{P}\left(X_{0}>h\right), \quad \forall k, h=0,1, \ldots
$$

Moreover,

$$
\sum_{h=0}^{\infty} \Gamma(h)=\lambda \mathrm{E}[X] \mathrm{E}\left[X_{0}\right]=\frac{\lambda}{2} \mathrm{E}[X(X+1)] .
$$

In the rest of the paper, we will make the following assumption on $\bar{F}$, the tail distribution of $X$ :

(A6) $\bar{F}(x)=\mathrm{P}(X>x) \sim x^{-\alpha} L(x), \quad$ with $\alpha>1$ and $L$ a slowly varying function.

We define by $\overline{F^{s}}$ the integrated tail distribution as follows:

$$
\overline{F^{s}}(x)=\min \left\{1, \int_{x}^{\infty} \bar{F}(u) d u\right\} .
$$

Thanks to the assumption on $F$, by Karamata's Theorem [4] the tail of $F^{s}$ is such that

$$
\overline{F^{s}}(x) \sim x^{-(\alpha-1)} L^{\prime}(x), \quad \text { with } L^{\prime} \text { a slowly varying function. }
$$

In this case we have the following important characteristics of the input process:

Proposition 2 [3,12]. Under the hypotheses (A1), (A2), (A3), (A4), (A5), and (A6) the stationary process $\left\{\sigma_{k}, k=0,1,2, \ldots\right\}$ is long-range dependent, that is

$$
\sum_{h=0}^{\infty} \Gamma(h)=\infty
$$

if and only if $1<\alpha<2$. Moreover, the process $\left\{\sigma_{k}, k=0,1,2, \ldots\right\}$ is asymptotically (second order) self-similar with Hurst parameter $H=\frac{3-\alpha}{2}$.

Now, thanks to (5), $\mathrm{E}\left[\sigma_{1}\right]=\gamma$, and then under the Loynes's stability condition $\gamma<a$ we have

$$
W_{\infty} \stackrel{\text { Law }}{=} \sup _{k \geqslant 1}\left[\sum_{k=1}^{\infty}\left(\sigma_{k}-a\right)\right]^{+},
$$

where the formula on the right is the solution of (3) when $W_{0}=0$, obtained thanks to the reversibility property of the process $\left\{\sigma_{k}, k=0,1,2, \ldots\right\}$.

\subsection{Exact asymptotics for the stationary workload}

Theorem 1 (Main Result). Under the assumption (A1), (A2), (A3), (A4), (A5), (A6) and

$$
\begin{aligned}
& \star \quad \gamma<a, \\
& \star \quad 1+\gamma>a,
\end{aligned}
$$

we have

$$
\mathrm{P}\left(W_{\infty}>x\right) \sim \frac{\lambda}{a-\gamma}(1+\gamma-a)^{\alpha-1} \overline{F^{s}}(x), \text { as } x \rightarrow \infty .
$$

We observe that the assumption $\gamma<a$ is the Loynes's stability condition. Moreover, since $\gamma$ is the mean number of customer in the $M / G / \infty$ queue, the assumption $1+\gamma>a$ says that the service system becomes unstable when one customer stays indefinitely in the queue. 


\section{Proof of the main result}

We briefly outline the argument of the proof. The main idea is to apply the main result of [2] to our framework. We first derive an upper bound $W \leqslant U+V$, where $U$ and $V$ are independent and $V$ is the stationary workload of a $G I / G I / 1 / \infty$ queue. This upper bound allows us to use Theorem 8 of [2], which shows that the probability $\mathrm{P}(W>x)$ is mainly due to one very large $X_{j, i}$ whereas the others remain close to their mean. Denote

$$
W=\sup _{k \geqslant 1}\left[\sum_{k=1}^{\infty}\left(\sigma_{k}-a\right)\right]^{+}
$$

\subsection{An upper bound and the single big event theorem}

We first derive an upper bound for the random variable $W$. We have

$$
\sum_{k=1}^{n} \sum_{i=1}^{b_{0}} 1_{\left\{X_{0, i}>k\right\}}=\sum_{i=1}^{b_{0}} \sum_{k=1}^{n} 1_{\left\{X_{0, i}>k\right\}}=\sum_{i=1}^{b_{0}}\left[X_{0, i} \wedge n\right], \quad \forall n \geqslant 1,
$$

and

$$
\sum_{k=1}^{n} \sum_{j=1}^{k} \sum_{i=1}^{b_{j}} 1_{\left\{X_{j, i}>k-j\right\}}=\sum_{j=1}^{n} \sum_{i=1}^{b_{j}} \sum_{k=1}^{n} 1_{\left\{X_{j, i}>k-j\right\}}=\sum_{j=1}^{n} \sum_{i=1}^{b_{j}}\left[X_{j, i} \wedge(n-j)\right], \quad \forall n \geqslant 1 .
$$

Hence

$$
\begin{aligned}
W & =\sup _{n \geqslant 1}\left[\sum_{i=1}^{b_{0}}\left(X_{0, i} \wedge n\right)+\sum_{j=1}^{n} \sum_{i=1}^{b_{j}}\left(X_{j, i} \wedge(n-j)\right)-n a\right]^{+}=\sup _{n \geqslant 1}\left(W_{n}\right) \leqslant \\
& \leqslant \sum_{i=1}^{b_{0}} X_{0, i}+\sup _{n \geqslant 1}\left[\sum_{j=1}^{n}\left(Y_{j}-a\right)\right]^{+}=U+V=Z,
\end{aligned}
$$

where

$$
U=\sum_{i=1}^{b_{0}} X_{0, i}, \quad Y_{j}=\sum_{i=1}^{b_{j}} X_{j, i}, \quad V=\sup _{n \geqslant 1}\left[\sum_{j=1}^{n}\left(Y_{j}-a\right)\right]^{+} .
$$

Note that $V$ is finite and corresponds to the stationary waiting time of a $D / G I / 1$ system with service times $Y_{j}$ with $\mathrm{E}\left[Y_{1}\right]=\gamma<a$.

Let $N_{x}$ be a function such that $N_{x} \uparrow \infty$ and $N_{x} \bar{F}(x)=o\left(\overline{F^{s}}(x)\right)$ whose existence is ensured by the heavy-tail property $[4,6,18]$. Using Theorem 8 of [2], we derive an asymptoptic equivalence of $\mathrm{P}(W>x)$ as $x \rightarrow \infty$, given by the formula (20).

Proposition 3. For any $x$, let $\left\{K_{n, x}, n \geqslant N_{x}\right\}$ be a sequence of events such that

a) for any $n \geqslant N_{x}$, the events $K_{n, x}$ and $Y_{n}$ are independent;

b) $\lim _{x \rightarrow \infty} \inf _{n \geqslant N_{x}} \mathrm{P}\left(K_{n, x}\right)=1$,

and define for any sequence $\eta_{n}$ tending to 0 , as $n \rightarrow \infty$,

$$
A_{n, x}=K_{n, x} \cap\left\{Y_{n}>x+n\left(a-\gamma+\eta_{n}\right)\right\} .
$$

Let $L_{x}$ be an event such that

c) the event $L_{x}$ and the random variable $U$ are independent;

d) $\lim _{x \rightarrow \infty} \mathrm{P}\left(L_{x}\right)=1$, 
and define

$$
B_{x}=\{U>x\} \cap L_{x}
$$

Then, as $x \rightarrow \infty$,

$$
\mathrm{P}(W>x)=\sum_{n \geqslant N_{x}} \mathrm{P}\left(W>x, A_{n, x}\right)+\mathrm{P}\left(W>x, B_{x}\right)+o\left(\overline{F^{s}}(x)\right)
$$

Proof. We note that $\mathrm{P}(U>x) \sim \lambda \overline{F^{s}}(x)[4,6,18]$ as $x \rightarrow \infty$, and thanks to Veraverbeke's Theorem $\mathrm{P}(V>x) \sim \frac{\lambda}{a-\gamma} \overline{F^{s}}(x)$ as $x \rightarrow \infty$. Moreover $U$ and $V$ are independent, and then

$$
\mathrm{P}(Z>x)=\mathrm{P}(U+V>x) \sim \lambda\left(1+\frac{1}{a-\gamma}\right) \overline{F^{s}}(x), x \rightarrow \infty .
$$

Let $K_{n, x}, L_{x}, A_{n, x}$ be events similar to the assumptions of this proposition. Denote $A_{x}=\cup_{n \geqslant N_{x}} A_{n, x}$ and $C_{x}=$ $=A_{x} \cup B_{x}$. Moreover we define $\tilde{K}_{n, x}=K_{n, x} \cap\{U \leqslant x\}$, and $\tilde{A}_{n, x}$ the corresponding events as in (18); and then $\tilde{A}_{x}$ and $\tilde{C}_{x}$. Evidently $\tilde{K}_{n, x}$ and $\tilde{A}_{n, x}$ satisfy again the assumptions a), b) of this proposition. Now the following bound holds:

$$
\mathrm{P}(Z>x) \geqslant \mathrm{P}\left(Z>x, C_{x}\right) \geqslant \mathrm{P}\left(V>x, \tilde{A}_{x}\right)+\mathrm{P}\left(B_{x}\right) .
$$

Now $V, \tilde{K}_{n, x}$, and $\tilde{A}_{n, x}$ satisfy the assumptions of the Corollary 2 of [2]. Therefore,

$$
\mathrm{P}\left(V>x, \tilde{A}_{x}\right)+o\left(\overline{F^{s}}(x)\right)=\mathrm{P}\left(V>x, A_{x}\right)+o\left(\overline{F^{s}}(x)\right)=\mathrm{P}(V>x) \sim \frac{\lambda}{a-\gamma} \overline{F^{s}}(x) .
$$

By the previous equality and since $\mathrm{P}\left(B_{x}\right)=\mathrm{P}(U>x) \mathrm{P}\left(L_{x}\right) \sim \lambda \overline{F^{s}}(x)$ as $x \rightarrow \infty$, we have from (22)

$$
\mathrm{P}(Z>x)=\mathrm{P}\left(Z>x, C_{x}\right)+o\left(\overline{F^{s}}(x)\right) .
$$

Now we have

$$
\mathrm{P}\left(W>x, C_{x}\right) \leqslant \mathrm{P}(W>x) \leqslant \mathrm{P}\left(W>x, C_{x}\right)+\mathrm{P}\left(Z>x, C_{x}^{c}\right)=\mathrm{P}\left(W>x, C_{x}\right)+o\left(\overline{F^{s}}(x)\right),
$$

where the last equality follows from (24). Then $\mathrm{P}(W>x)=\mathrm{P}\left(W>x, C_{x}\right)+o\left(\overline{F^{s}}(x)\right)$. Now we can construct disjoint events $K_{n, x}^{\prime}$ for any $n \geqslant N_{x}$ that satisfy the assumptions $a$ ), $b$ ), using similar arguments of Corollary 2 of [2]. Then

$$
\mathrm{P}\left(W>x, C_{x}^{\prime}\right)=\sum_{n \geqslant N_{x}} \mathrm{P}\left(W>x, A_{n, x}^{\prime}\right)+\mathrm{P}\left(W>x, B_{x}\right)+o\left(\overline{F^{s}}(x)\right) .
$$

Moreover, it is not difficult to prove that

$$
\sum_{n \geqslant N_{x}} \mathrm{P}\left(W>x, A_{n, x}^{\prime}\right)-\sum_{n \geqslant N_{x}} \mathrm{P}\left(W>x, A_{n, x}\right)=o\left(\overline{F^{s}}(x)\right)
$$

and then the equivalence (20) follows. See [18] for details.

\subsection{Asymptotic equivalence for $\mathrm{P}\left(W>x, B_{x}\right)$}

Proposition 4. Under the previous assumptions, we have

$$
\lim _{x \rightarrow \infty} \frac{\mathrm{P}\left(W>x, B_{x}\right)}{\overline{F^{s}}(x)}=\lambda(1+\gamma-a)^{\alpha-1}
$$

We will prove this proposition in two steps. First we derive a lower bound and then an upper bound. 


\subsubsection{Lower bound}

We take

$$
L_{x}=\left\{\frac{\sum_{j=1}^{m} \sum_{i=1}^{b_{j}}\left(X_{j, i} \wedge(m-j)\right)}{m}>\gamma-\varepsilon_{m}, \forall m>x\right\} .
$$

By definition, $L_{x}$ satisfies the assumption c) of Proposition 3, and d) thanks to Lemma 1 for some sequence $\varepsilon_{n}$ such that $\varepsilon_{n} \downarrow 0$ and $n \varepsilon_{n} \rightarrow \infty$ as $n \rightarrow \infty$. We observe that for all $n$ we have

$$
W \geqslant \sum_{i=1}^{b_{0}}\left(X_{0, i} \wedge n\right)+\sum_{j=1}^{n} \sum_{i=1}^{b_{j}}\left(X_{j, i} \wedge(n-j)\right)-n a .
$$

Taking $n=U=\sum_{i=1}^{b_{0}} X_{0, i}$, we have

$$
W \geqslant U+\sum_{j=1}^{U} \sum_{i=1}^{b_{j}}\left(X_{j, i} \wedge(U-j)\right)-U a=U(1-a)+\sum_{j=1}^{U} \sum_{i=1}^{b_{j}}\left(X_{j, i} \wedge(U-j)\right) .
$$

On the event $B_{x}=\left\{U>x, L_{x}\right\}$ we have

$$
W \geqslant U(1-a)+U\left(\gamma-\varepsilon_{U}\right)=U\left(1+\gamma-a-\varepsilon_{U}\right) .
$$

We recall that $\varepsilon_{n} \downarrow 0$ as $n \rightarrow \infty$ and that the condition $1+\gamma-a>0$ is assumed. Let $\delta>0$ be such that $1+\gamma-a-\delta>0$. For $n \geqslant n_{0}$ with $n_{0}$ large enough, $\varepsilon_{n}<\delta$; therefore, for $x$ large enough, if $U>x \geqslant n_{0}$ we have $\varepsilon_{U}<\varepsilon_{x}<\delta$. Moreover, since $\gamma<a$, we have also $1+\gamma-a<1$. Hence

$$
\begin{aligned}
\mathrm{P}\left(W>x, B_{x}\right) & \geqslant \mathrm{P}\left(U\left(1+\gamma-a-\varepsilon_{x}\right)>x, U>x, L_{x}\right)= \\
& =\mathrm{P}\left(U\left(1+\gamma-a-\varepsilon_{x}\right)>x\right) \mathrm{P}\left(L_{x}\right)= \\
& \geqslant \mathrm{P}\left(U>\frac{x}{1+\gamma-a-\delta}\right) \mathrm{P}\left(L_{x}\right) .
\end{aligned}
$$

Now since $\mathrm{P}(U>x) \sim \lambda \overline{F^{s}}(x)$ as $x \rightarrow \infty[4,6,18], \overline{F^{s}}(x) \sim x^{-(\alpha-1)} L(x)$ with $L$ a slowly varying function, and finally sending $\delta$ to 0 , we conclude that

$$
\liminf _{x \rightarrow \infty} \frac{\mathrm{P}\left(W>x, B_{x}\right)}{\overline{F^{s}}(x)} \geqslant \lambda(1+\gamma-a)^{\alpha-1} .
$$

\subsubsection{Upper bound}

Let $\alpha_{x}=x^{\frac{1}{2}-\varepsilon}$ for some $0<\varepsilon<\frac{1}{2}$. Next take

$$
\begin{aligned}
& R_{x}=\left\{\sum_{j=1}^{n}\left(\sum_{i=1}^{b_{j}}\left(X_{j, i} \wedge(n-j)\right)-\gamma\right) \leqslant n \varepsilon_{n}, \forall n \geqslant \alpha_{x}\right\}, \\
& Q_{x}=\left\{\sum_{j=1}^{n}\left(\sum_{i=1}^{b_{j}}\left(X_{j, i} \wedge(n-j)\right)-a\right) \leqslant \alpha_{x}, \forall n<\alpha_{x}\right\} .
\end{aligned}
$$

Define

$$
L_{x}=R_{x} \cap Q_{x} .
$$

Observe that $L_{x}$ satisfies the assumptions of Proposition 3. In fact, for all $n$

$$
\sum_{j=1}^{n}\left(\sum_{i=1}^{b_{j}}\left(X_{j, i} \wedge(n-j)\right)-a\right) \leqslant \sum_{j=1}^{n}\left(Y_{j}-a\right) \leqslant V<\infty \text { a.s. }
$$


Therefore $\mathrm{P}\left(Q_{x}\right) \rightarrow 1$, as $x \rightarrow \infty$. Moreover $\mathrm{P}\left(R_{x}\right) \rightarrow 1$ as $x \rightarrow \infty$ thanks to Lemma 1 . Now we use Lemma 2, with $N=b_{0}, Z_{i}=X_{i, 0}$, we have $S_{N}=U$. Moreover, we set $M_{0} \triangleq M_{N}$ and $M_{0}^{1} \triangleq M_{N}^{1}$, so that

$$
\mathrm{P}\left(W>x, B_{x}\right)=\mathrm{P}\left(W>x, U>x, L_{x}\right)=\mathrm{P}\left(W>x, M_{0}>x, M_{0}^{1} \leqslant \alpha_{x}, L_{x}\right)+o\left(\overline{F^{s}}(x)\right) .
$$

We have that

$$
W=\sup _{n \geqslant 1} W_{n} \leqslant \max \left\{\sup _{n \leqslant \alpha_{x}} W_{n}, \sup _{n>\alpha_{x}} W_{n}\right\} .
$$

Let $\delta>0$ such that $\gamma-a+\delta<0$. Since $\varepsilon_{n} \downarrow 0$, there exists an $n_{0}$ such that for all $n \geqslant n_{0}, \varepsilon_{n}<\delta$. Hence, if $x$ is large enough, for $n>\alpha_{x} \geqslant n_{0}, \varepsilon_{n}<\delta$. Now on the event $\left\{M_{0}>x, M_{0}^{1} \leqslant \alpha_{x}\right\}$, for all $n$ we have

$$
\sum_{i=1}^{b_{0}}\left(X_{0, i} \wedge n\right) \leqslant M_{0} \wedge n+b_{0}\left(\alpha_{x} \wedge n\right)
$$

Again, on the event $\left\{M_{0}>x, M_{0}^{1} \leqslant \alpha_{x}, L_{x}\right\}$, we have

$$
\begin{aligned}
W & \leqslant \max \left\{\sup _{n \leqslant \alpha_{x}}\left[M_{0} \wedge n+b_{0}\left(\alpha_{x} \wedge n\right)+\alpha_{x}\right]^{+}, \sup _{n>\alpha_{x}}\left[M_{0} \wedge n+b_{0}\left(\alpha_{x} \wedge n\right)+n(\gamma-a+\delta)\right]^{+}\right\} \leqslant \\
& \leqslant \max \left\{\left[\alpha_{x}+b_{0} \alpha_{x}+\alpha_{x}\right], M_{0}(1+\gamma-a+\delta)+b_{0} \alpha_{x}\right\} \leqslant \\
& \leqslant M_{0}(1+\gamma-a+\delta)+\left(b_{0}+2\right) \alpha_{x},
\end{aligned}
$$

since for $x$ large enough $x>\alpha_{x}$ and by assumption $1+\gamma-a>0$. Therefore,

$$
\mathrm{P}\left(W>x, B_{x}\right) \leqslant \mathrm{P}\left(M_{0}>\frac{x}{1+\gamma-a+\delta}-\frac{\left(2+b_{0}\right) \alpha_{x}}{1+\gamma-a+\delta}\right) .
$$

Let $c_{\delta}=(1+\gamma-a+\delta)^{-1}$. Then by definition of $M_{0}$

$$
\mathrm{P}\left(W>x, B_{x}\right) \leqslant \sum_{n=1}^{\infty} n \mathrm{P}\left(b_{0}=n\right) \mathrm{P}\left(X_{0}>c_{\delta} x-c_{\delta}(n+2) \alpha_{x}\right) .
$$

For any continuous, positive real function $h$ we can write

$$
\mathrm{P}\left(W>x, B_{x}\right) \leqslant \sum_{n=1}^{h(x)} n \mathrm{P}\left(b_{0}=n\right) \mathrm{P}\left(X_{0}>c_{\delta} x-c_{\delta}(n+2) \alpha_{x}\right)+\sum_{n>h(x)} n \mathrm{P}\left(b_{0}=n\right) .
$$

Now let $h(x)=\frac{\sqrt{x}}{c_{\delta}}-2$, for $x$ large; then $c_{\delta}(2+n) \alpha_{x} \leqslant c_{\delta}(2+h(x)) \alpha_{x}=x^{1-\varepsilon}$, for $n \leqslant h(x)$. Moreover

$$
\sum_{n>h(x)} n \mathrm{P}\left(b_{0}=n\right)=\gamma \mathrm{P}\left(b_{0} \geqslant h(x)\right) \leqslant A e^{-h(x)},
$$

where $A$ is some positive constant. Therefore,

$$
\begin{aligned}
\mathrm{P}\left(W>x, B_{x}\right) & \leqslant \mathrm{P}\left(X_{0}>c_{\delta} x-x^{1-\varepsilon}\right) \sum_{n=1}^{\infty} n \mathrm{P}\left(b_{0}=n\right)+A e^{-h(x)}= \\
& =\mathrm{P}\left(X_{0}>c_{\delta} x-x^{1-\varepsilon}\right) \lambda \mathrm{E}[X]+A e^{-h(x)} .
\end{aligned}
$$

Now divide both members by $\overline{F^{s}}(x)$. We have $A e^{-h(x)}=o\left(\overline{F^{s}}(x)\right)$. In fact, since $\overline{F^{s}}(x) \sim L(x) x^{-(\alpha-1)}$ as $x \rightarrow \infty$ for some $L$ slowly varying function, we have

$$
\log \left(\frac{A e^{-h(x)}}{\overline{F^{s}}(x)}\right)=\log (A)-h(x)-\log (L(x))+(\alpha-1) \log (x) \rightarrow-\infty,
$$


recalling the representation theorem for the slowly varying function $L$ [4]. Now we observe that $\mathrm{P}\left(X_{0}>x\right) \sim$ $\sim(\mathrm{E}[X])^{-1} \overline{F^{s}}(x)$. Moreover, since $x^{1-\varepsilon}=o(x), \overline{F^{s}}\left(c_{\delta} x-x^{1-\varepsilon}\right) \sim \overline{F^{s}}\left(c_{\delta} x\right) \sim c_{\delta}^{-(\alpha-1)} \overline{F^{s}}(x)$, as $x \rightarrow \infty$. Therefore, sending $\delta$ to zero, $c_{\delta} \rightarrow(1+\gamma-a)^{-1}$ and then we obtain

$$
\limsup _{x \rightarrow \infty} \frac{\mathrm{P}\left(W>x, B_{x}\right)}{\overline{F^{s}}(x)} \leqslant \lambda(1+\gamma-a)^{\alpha-1} .
$$

Therefore by (27) and (29), the proposition 4 is proved.

\subsection{Asymptotic equivalence for $\sum_{n \geqslant N_{x}} \mathrm{P}\left(W>x, A_{n, x}\right)$}

Proposition 5. Under the previous assumptions

$$
\lim _{x \rightarrow \infty} \frac{\sum_{n \geqslant N_{x}} \mathrm{P}\left(W>x, A_{n, x}\right)}{\overline{F^{s}}(x)}=\frac{\lambda(1+\gamma-a)}{a-\gamma}(1+\gamma-a)^{\alpha-1} .
$$

We will prove this proposition in two steps. First we derive a lower bound and then an upper bound.

\subsubsection{Lower bound}

We take $\forall n \geqslant N_{x}$

$$
K_{n, x}=\left\{\frac{\sum_{j=1}^{n-1} \sum_{i=1}^{b_{j}}\left(X_{j, i} \wedge(k-j)\right)+\sum_{j=n+1}^{k} \sum_{i=1}^{b_{j}}\left(X_{j, i} \wedge(k-j)\right)}{k}-\gamma \geqslant-\eta_{k}, \quad \forall k \geqslant n\right\},
$$

with some sequence $\eta_{n}$ such that $\eta_{n} \downarrow 0$ and $n \eta_{n} \rightarrow \infty$ as $n \rightarrow \infty$. Hence $K_{n, x}$ satisfies the hypothesis a) of Proposition 3 and b) thanks to lemma 1 .

We have for all $k \geqslant n$,

$$
W \geqslant Y_{n} \wedge(k-n)+\sum_{j=1}^{n-1} \sum_{i=1}^{b_{j}}\left(X_{j, i} \wedge(k-j)\right)+\sum_{j=n+1}^{k} \sum_{i=1}^{b_{j}}\left(X_{j, i} \wedge(k-j)\right)-k a .
$$

On the event $K_{n, x}$, taking $k=n+Y_{n}$, we have

$$
W \geqslant Y_{n}+\left(n+Y_{n}\right)\left(\gamma-a-\eta_{n}\right)=Y_{n}\left(1+\gamma-a-\eta_{n}\right)+n\left(\gamma-a-\eta_{n}\right) .
$$

Since $1+\gamma-a<1$, we have

$$
\sum_{n \geqslant N_{x}} \mathrm{P}\left(W>x, A_{n, x}\right) \geqslant \sum_{n \geqslant N_{x}} \mathrm{P}\left(Y_{n}\left(1+\gamma-a-\eta_{n}\right) \geqslant x+n\left(a-\gamma+\eta_{n}\right)\right) \mathrm{P}\left(K_{n, x}\right) .
$$

Let $\delta>0$ such that $1+\gamma-a-\delta>0$. Since $\eta_{n} \downarrow 0$ as $n \rightarrow \infty$, there exists $n_{0}$ such that for all $n \geqslant n_{0}, \eta_{n}<\delta$. Recalling that $N_{x} \rightarrow \infty$ as $x \rightarrow \infty$, if $x$ is large enough, and for all $n \geqslant N_{x} \geqslant n_{0}$ we have

$$
\sum_{n \geqslant N_{x}} \mathrm{P}\left(W>x, A_{n, x}\right) \geqslant \sum_{n \geqslant N_{x}} \mathrm{P}\left(Y_{n}(1+\gamma-a-\delta) \geqslant x+n(a-\gamma+\delta)\right) \mathrm{P}\left(K_{n, x}\right) .
$$

Therefore

$$
\liminf _{x \rightarrow \infty} \frac{\sum_{n \geqslant N_{x}} \mathrm{P}\left(W>x, A_{n, x}\right)}{\overline{F^{s}}(x)} \geqslant \lambda \frac{(1+\gamma-a-\delta)}{a-\gamma+\delta}(1+\gamma-a-\delta)^{\alpha-1},
$$

where the formula on the right follows thanks to assumption b) of Proposition 3,the subexponentiality of $Y_{1}$, and since $N_{x} \bar{F}(x)=o\left(\overline{F^{s}}(x)\right)[2,18]$.

Finally, sending $\delta$ to zero, we obtain

$$
\liminf _{x \rightarrow \infty} \frac{\sum_{n \geqslant N_{x}} \mathrm{P}\left(W>x, A_{n, x}\right)}{\overline{F^{s}}(x)} \geqslant \lambda \frac{(1+\gamma-a)}{a-\gamma}(1+\gamma-a)^{\alpha-1} .
$$




\subsubsection{Upper bound}

We choose a function $\alpha_{x}$ such that $\alpha_{x} \uparrow \infty, \alpha_{x}=o(x)$ as $x \rightarrow \infty$. Therefore, $\overline{F^{s}}\left(x-\alpha_{x}\right) \sim \overline{F^{s}}(x)$ as $x \rightarrow \infty$. Define $U_{k}=\left[\sum_{j=1}^{k} \sum_{i=1}^{b_{j}}\left(X_{j, i} \wedge(k-j)\right)-k a\right]^{+}$. For any $n$ fixed

$$
\begin{aligned}
W \leqslant U+\max \left\{\sup _{1 \leqslant k \leqslant n-1} U_{k}, \sup _{k \geqslant n}\left[\sum _ { j = 1 } ^ { n - 1 } \sum _ { i = 1 } ^ { b _ { j } } \left(X_{j, i} \wedge\right.\right.\right. & (k-j))+ \\
& \left.\left.+\sum_{i=1}^{b_{n}}\left(X_{n, i} \wedge(k-n)\right)+\sum_{j=n+1}^{k} \sum_{i=1}^{b_{j}}\left(X_{j, i} \wedge(k-j)\right)-k a\right]^{+}\right\} .
\end{aligned}
$$

Now we note that $\forall n \in \mathbb{N}$, $\sup _{1 \leqslant k \leqslant n-1} U_{k} \leqslant V<\infty$, a.s.; then

$$
\lim _{x \rightarrow \infty} \mathrm{P}\left(\sup _{1 \leqslant k \leqslant n-1} U_{k}<\alpha_{x}\right)=1 .
$$

For all $n \geqslant N_{x}$ define

$$
K_{n, x}^{\prime}=\left\{\sum_{j=1}^{n-1} \sum_{i=1}^{b_{j}}\left(X_{j, i} \wedge(k-j)\right)+\sum_{j=n+1}^{k} \sum_{i=1}^{b_{j}}\left(X_{j, i} \wedge(k-j)\right)-k \gamma<k \eta_{k}, \forall k \geqslant n\right\},
$$

where $\eta_{n}$ is a some sequence such that $\eta_{n} \downarrow 0, n \eta_{n} \rightarrow \infty$ as $n \rightarrow \infty$. Now define $\forall n \geqslant N_{x}$

$$
K_{n, x}=K_{n, x}^{\prime} \cap\left\{U \leqslant \alpha_{x}\right\} \cap\left\{\sup _{1 \leqslant k \leqslant n-1} U_{k} \leqslant \alpha_{x}\right\},
$$

which is independent of $Y_{n}$ and satisfies also the assumption b) of Proposition 3.

Thanks to the Lemma 3 with $Y_{n}=S_{n}$ we have

$$
\begin{aligned}
& \sum_{n \geqslant N_{x}} \mathrm{P}\left(W>x, K_{n, x}, Y_{n}>x+n\left(a-\gamma+\eta_{n}\right)\right)= \\
& =\sum_{n \geqslant N_{x}} \mathrm{P}\left(W>x, K_{n, x}, M_{n}>x+n\left(a-\gamma+\eta_{n}\right), M_{n}^{1} \leqslant \alpha_{x}\right)+o\left(\overline{F^{s}}(x)\right) .
\end{aligned}
$$

We observe that on the event $\left\{M_{n}>x+n\left(a-\gamma+\eta_{n}\right), M_{n}^{1} \leqslant \alpha_{x}\right\}$ we have

$$
\sum_{i=1}^{b_{n}}\left(X_{n, i} \wedge(k-n)\right) \leqslant M_{n} \wedge(k-n)+b_{n}\left(\alpha_{x} \wedge(k-n)\right) ;
$$

moreover, on the event $\left\{M_{n}>x+n\left(a-\gamma+\eta_{n}\right), M_{n}^{1} \leqslant \alpha_{x}, K_{n, x}\right\}$ we have

$$
W \leqslant \alpha_{x}+\max \left(\alpha_{x}, \sup _{k \geqslant n}\left[M_{n} \wedge(k-n)+b_{n}\left(\alpha_{x} \wedge(k-n)\right)+k\left(\gamma-a+\eta_{k}\right)\right]^{+}\right) .
$$

Now let $\delta>0$ such that $\gamma-a+\delta<0$. We have $\eta_{k}<\delta$, for all $k \geqslant n \geqslant N_{x}$, for $x$ large enough. Hence

$$
W \leqslant \alpha_{x}+\max \left(\alpha_{x}, M_{n}(1+\gamma-a+\delta)+n(\gamma-a+\delta)+b_{n} \alpha_{x}\right),
$$

since $-1<\gamma-a+\delta<0$. Therefore

$$
\begin{aligned}
& \sum_{n \geqslant N_{x}} \mathrm{P}\left(W>x, K_{n, x}, Y_{n}>x+n\left(a-\gamma+\eta_{n}\right)\right) \leqslant \\
\leqslant & \sum_{n \geqslant N_{x}} \mathrm{P}\left(M_{n}(1+\gamma-a+\delta)+n(\gamma-a+\delta)+b_{n} \alpha_{x}>x\right)= \\
= & \sum_{n \geqslant N_{x}} \sum_{k=0}^{\infty} \mathrm{P}\left(b_{n}=k\right) \mathrm{P}\left(\max _{i=1}^{k} X_{n, i}(1+\gamma-a+\delta)>x-k \alpha_{x}+n(a-\gamma-\delta)\right) .
\end{aligned}
$$


With $c_{\delta}=(1+\gamma-a+\delta)^{-1}$ and $d_{\delta}=\frac{a-\gamma-\delta}{1+\gamma-a+\delta}$, we have

$$
\begin{aligned}
& \leqslant \sum_{n \geqslant N_{x}} \sum_{k=1}^{\infty} \mathrm{P}\left(b_{1}=k\right) \mathrm{P}\left(\max _{i=1}^{k} X_{i}>c_{\delta} x-c_{\delta} k \alpha_{x}+n d_{\delta}\right) \leqslant \\
& \leqslant \sum_{n \geqslant N_{x}} \sum_{k=1}^{\infty} k \mathrm{P}\left(b_{1}=k\right) \mathrm{P}\left(X>c_{\delta} x-c_{\delta} k \alpha_{x}+n d_{\delta}\right)= \\
& =\frac{1}{d_{\delta}} \sum_{k=1}^{\infty} \mathrm{P}\left(b_{1}=k\right) k \overline{F^{s}}\left(c_{\delta} x-c_{\delta} k \alpha_{x}\right) .
\end{aligned}
$$

With similar arguments used for the upper bound of $\mathrm{P}\left(W>x, B_{x}\right)$ we have that

$$
\limsup _{x \rightarrow \infty} \frac{\sum_{n>N_{x}} \mathrm{P}\left(W>x, A_{n, x}\right)}{\overline{F^{s}}(x)} \leqslant \lambda \frac{1+\gamma-a+\delta}{a-\gamma-\delta}(1+\gamma-a+\delta)^{\alpha-1} .
$$

Finally sending $\delta$ to zero, we conclude that

$$
\limsup _{x \rightarrow \infty} \frac{\sum_{n \geqslant N_{x}} \mathrm{P}\left(W>x, A_{n, x}\right)}{\overline{F^{s}}(x)} \leqslant \lambda \frac{(1+\gamma-a)}{a-\gamma}(1+\gamma-a)^{\alpha-1},
$$

which ends the proof of Proposition 5.

\section{Appendix}

Lemma 1. Under the hypotheses (A1),(A2), and (A3), we have

$$
\frac{1}{n} \sum_{j=1}^{n} \sum_{i=1}^{b_{j}}\left(X_{j, i} \wedge(n-j)\right) \rightarrow \gamma \quad \text { a.s. as } n \rightarrow \infty .
$$

Proof. By the strong law of large numbers (SLLN)

$$
\limsup _{n \rightarrow \infty} \frac{1}{n} \sum_{j=1}^{n}\left(\sum_{i=1}^{b_{j}}\left(X_{j, i} \wedge(n-j)\right)-\gamma\right) \leqslant \frac{1}{n} \sum_{j=1}^{n}\left(\sum_{i=1}^{b_{j}} X_{j, i}-\gamma\right) \leqslant 0, \text { a.s. }
$$

Now $\forall M<n$,

$$
\begin{aligned}
& \frac{1}{n} \sum_{j=1}^{n}\left(\sum_{i=1}^{b_{j}}\left(X_{j, i} \wedge(n-j)\right)-\gamma\right) \geqslant \\
& \geqslant \frac{1}{n} \sum_{j=1}^{n-M}\left(\sum_{i=1}^{b_{j}}\left(X_{j, i} \wedge(n-j)\right)-\lambda \mathrm{E}[X]\right)-\frac{M \lambda \mathrm{E}[X]}{n} \geqslant \\
& \geqslant \frac{1}{n} \sum_{j=1}^{n-M}\left(\sum_{i=1}^{b_{j}}\left(X_{j, i} \wedge M\right)-\lambda \mathrm{E}[X \wedge M]\right)+(n-M)\left(\frac{\lambda \mathrm{E}[X \wedge M]-\lambda \mathrm{E}[X]}{n-M}\right)-\frac{M \lambda \mathrm{E}[X]}{n} .
\end{aligned}
$$

Therefore, again by the SLLN, for any $M$

$$
\liminf _{n \rightarrow \infty} \frac{1}{n} \sum_{j=1}^{n}\left(\sum_{i=1}^{b_{j}}\left(X_{j, i} \wedge(n-j)\right)-\gamma\right) \geqslant \lambda \mathrm{E}[X \wedge M]-\lambda \mathrm{E}[X], \text { a.s. }
$$

Since as $M \rightarrow \infty$, it holds that $\mathrm{E}[X \wedge M] \uparrow \mathrm{E}[X]$,

$$
\liminf _{n \rightarrow \infty} \frac{1}{n} \sum_{j=1}^{n}\left(\sum_{i=1}^{b_{j}}\left(X_{j, i} \wedge(n-j)\right)-\gamma\right) \geqslant 0, \text { a.s. }
$$

and the lemma is proved. 
Lemma 2. Let $N$ be a Poisson random variable, and $\left\{Z_{i}\right\}_{i \in \mathbb{N}}$ be a sequence of i.i.d sub-exponential random variables with distribution function $G$. Let

$$
S_{N}=\sum_{i=1}^{N} Z_{i} \quad \text { and }, \quad M_{N}=\max _{i=1}^{N} Z_{i}
$$

Moreover let $i_{0}=\arg \max _{i=1}^{N} Z_{i}$ and $M_{N}^{1}=\max _{i=1, i \neq i_{0}}^{N} Z_{i}$. Then for any given event $E$ and for any function $\alpha_{x} \uparrow \infty$ as $x \rightarrow \infty$ such that $\alpha_{x}=o(x)$, we have

$$
\begin{aligned}
\mathrm{P}\left[E, S_{N}>x\right] & =\mathrm{P}\left[E, M_{N}>x\right]+o(\bar{G}(x))= \\
& =\mathrm{P}\left[E, M_{N}>x, M_{N}^{1} \leqslant \alpha_{x}\right]+o(\bar{G}(x)) .
\end{aligned}
$$

Proof. Let $p_{n}=\mathrm{P}(N=n)$. Since $\alpha_{x} \leqslant x$ for $x$ large,

$$
\frac{\mathrm{P}\left[M_{N}>x, M_{N}^{1} \leqslant \alpha_{x}\right]}{\bar{G}(x)}=\frac{\sum_{n=1}^{\infty} p_{n} \sum_{k=1}^{n} \mathrm{P}\left[Y_{k}>x, Y_{i} \leqslant \alpha_{x} \forall i \neq k\right]}{\bar{G}(x)}=\sum_{n=1}^{\infty} n p_{n} G\left(\alpha_{x}\right)^{n-1} .
$$

By the dominated convergence theorem,

$$
\lim _{x \rightarrow \infty} \frac{\mathrm{P}\left[M_{N}>x, M_{N}^{1} \leqslant \alpha_{x}\right]}{\bar{G}(x)}=\sum_{n=1}^{\infty} n p_{n}=\mathrm{E}[N] .
$$

Therefore, since

$$
\frac{\lim _{x \rightarrow \infty} \mathrm{P}\left(M_{N}>x\right)}{\bar{G}(x)}=\mathrm{E}[N]
$$

it follows from (38) that

$$
\mathrm{P}\left(M_{N}>x, M_{N}^{1}>\alpha_{x}\right)=o(\bar{G}(x)) .
$$

Since $M_{N} \leqslant S_{N}$, we have clearly $\mathrm{P}\left[E, S_{N}>x\right] \geqslant \mathrm{P}\left[E, M_{N}>x, M_{N}^{1} \leqslant \alpha_{x}\right]$. Moreover, we have

$$
\mathrm{P}\left[E, S_{N}>x\right] \leqslant \mathrm{P}\left[E, M_{N}>x, M_{N}^{1} \leqslant \alpha_{x}\right]+\mathrm{P}\left[M_{N}>x, M_{N}^{1}>\alpha_{x}\right]+\mathrm{P}\left[S_{N}>x, M_{N} \leqslant x\right] .
$$

By the subexponentiality $[4,6,18]$, we have $\mathrm{P}\left[S_{N}>x\right] \sim \mathrm{P}\left[M_{N}>x\right] \sim \mathrm{E}[N] \bar{G}(x), x \rightarrow \infty$. Hence

$$
\mathrm{P}\left[S_{N}>x, M_{N} \leqslant x\right]=\mathrm{P}\left[S_{N}>x\right]-\mathrm{P}\left[M_{N}>x\right]=o(\bar{G}(x)) .
$$

Finally by (39) and (40),

$$
\mathrm{P}\left(E, S_{N}>x\right)=\mathrm{P}\left(E, M_{N}>x, M_{N}^{1} \leqslant \alpha_{x}\right)+o(\bar{G}(x)) .
$$

Lemma 3. Under the conditions (A1), (A2), and (A3) define

$$
S_{n}=\sum_{i=1}^{b_{n}} X_{n, i}, \quad M_{n}=\max _{i=1}^{b_{n}} X_{n, i}, \quad i_{n}=\arg \max _{i=1, \cdots, b_{n}} X_{n, i}, \quad M_{n}^{1}=\max _{i \neq i_{n}, i=1}^{b_{n}} X_{n, i} .
$$

Then, $\forall c>0, \eta_{n} \downarrow 0$, as $n \rightarrow \infty$, for all events $E_{n}$ and for any function $\alpha_{x} \uparrow \infty$ as $x \rightarrow \infty$ with $\alpha_{x}=o(x)$, we have

$$
\sum_{n \geqslant N_{x}} \mathrm{P}\left(E_{n}, S_{n}>x+n\left(c+\eta_{n}\right)\right)=\sum_{n \geqslant N_{x}} \mathrm{P}\left(E_{n}, M_{n}>x+n\left(c+\eta_{n}\right), M_{n}^{1} \leqslant \alpha_{x}\right)+o\left(\overline{F^{s}}(x)\right) .
$$

Proof. We omit in the following proof the sequence $\eta_{n}$, but the same arguments are true, including $\eta_{n}$. For $x$ large enough

$$
\begin{aligned}
\sum_{n \geqslant 1} \mathrm{P}\left(M_{n}>x+n c, M_{n}^{1} \leqslant \alpha_{x}\right) & =\sum_{n \geqslant 1} \sum_{k=1}^{\infty} \mathrm{P}\left(b_{n}=k\right) \sum_{i=1}^{k} \mathrm{P}\left(X_{n, i}>x+n c, X_{n, j} \leqslant \alpha_{x} \forall j \neq i\right)= \\
& =\sum_{n \geqslant 1} \mathrm{P}(X>x+n c)\left(\sum_{k=1}^{\infty} \mathrm{P}\left(b_{1}=k\right) k\left[\mathrm{P}\left(X \leqslant \alpha_{x}\right)\right]^{k-1}\right) .
\end{aligned}
$$


Therefore by the dominated convergence theorem, we have

$$
\lim _{x \rightarrow \infty} \frac{\sum_{n \geqslant 1} \mathrm{P}\left(M_{n}>x+n c, M_{n}^{1} \leqslant \alpha_{x}\right)}{\overline{F^{s}}(x)}=\frac{1}{c} \sum_{k=1}^{\infty} \mathrm{P}\left(b_{1}=k\right) k=\frac{\lambda}{c} .
$$

On the other hand,

$$
\sum_{n \geqslant 1} \mathrm{P}\left(M_{n}>x+n c\right)=\sum_{n \geqslant 1} \mathrm{P}\left(M_{n}>x+n c, M_{n}^{1} \leqslant \alpha_{x}\right)+\sum_{n \geqslant 1} \mathrm{P}\left(M_{n}>x+n c, M_{n}^{1}>\alpha_{x}\right) .
$$

By the definition of $M_{n}$, we have also

$$
\lim _{x \rightarrow \infty} \frac{\sum_{n \geqslant 1} \mathrm{P}\left(M_{n}>x+n c\right)}{\overline{F^{s}}(x)}=\frac{\lambda}{c} .
$$

Therefore, by (41) and (42),

$$
\sum_{n \geqslant 1} \mathrm{P}\left(M_{n}>x+n c, M_{n}^{1}>\alpha_{x}\right)=o\left(\overline{F^{s}}(x)\right), x \rightarrow \infty .
$$

Moreover, since $S_{n} \geqslant M_{n}$,

$$
\sum_{n \geqslant 1} \mathrm{P}\left(E_{n}, S_{n}>x+n c\right) \geqslant \sum_{n \geqslant 1} \mathrm{P}\left(E_{n}, M_{n}>x+n c, M_{n}^{1} \leqslant \alpha_{x}\right) .
$$

Again

$$
\begin{aligned}
\sum_{n \geqslant 1} \mathrm{P}\left(E_{n}, S_{n}>x+n c\right) \leqslant & \sum_{n \geqslant 1} \mathrm{P}\left(E_{n}, M_{n}>x+n c, M_{n}^{1} \leqslant \alpha_{x}\right)+\sum_{n \geqslant 1} \mathrm{P}\left(M_{n}>x+n c, M_{n}^{1}>\alpha_{x}\right)+ \\
& +\sum_{n \geqslant 1} \mathrm{P}\left(S_{n}>x+n c, M_{n} \leqslant x+n c\right) .
\end{aligned}
$$

Now by the subexponentiality $[2,18]$, we have

$$
\sum_{n \geqslant 1} \mathrm{P}\left(S_{n}>x+n c\right) \sim \sum_{n \geqslant 1} \mathrm{P}\left(M_{n}>x+n c\right) \sim \lambda c^{-1} \overline{F^{s}}(x), x \rightarrow \infty .
$$

Hence

$$
\sum_{n \geqslant 1} P\left(S_{n}>x+n c, M_{n} \leqslant x+n c\right)=o\left(\overline{F^{s}}(x)\right) .
$$

Therefore, by (43) and (44),

$$
\sum_{n \geqslant 1} \mathrm{P}\left(E_{n}, S_{n}>x+n c\right)=\sum_{n \geqslant 1} \mathrm{P}\left(E_{n}, M_{n}>x+n c, M_{n}^{1} \leqslant \alpha_{x}\right)+o\left(\overline{F^{s}}(x)\right) .
$$

We conclude, observing that

$$
\begin{aligned}
& \sum_{n=1}^{N_{x}} \mathrm{P}\left(E_{n}, S_{n}>x+n c\right) \leqslant N_{x} \mathrm{P}\left(S_{1}>x\right)=o\left(\overline{F^{s}}(x)\right), \\
& \sum_{n=1}^{N_{x}} \mathrm{P}\left(E_{n}, M_{n}>x+n c, M_{n}^{1} \leqslant \alpha_{x}\right) \leqslant N_{x} \mathrm{P}\left(M_{1}>x\right)=o\left(\overline{F^{s}}(x)\right) .
\end{aligned}
$$




\section{References}

1. F. Baccelli and P. Brémaud, Elements of Queueing Theory, Applications of Mathematics, Vol. 26, SpringerVerlag, Berlin, (2003).

2. F. Baccelli and S. Foss, "Moments and tails in monotone-separable stochastic networks," Ann. Appl. Probab., 14, No. 2, 612-650 (2004).

3. D. Cox, "Long-range dependence: A review," in: Statistics: An Appraisal, H. A. David and H. T. David (eds.), The Iowa State (1984), pp. 55-74.

4. P. Embrechts, C. Klüppelberg, and T. Mikosch, Modelling Extremal Events, Applications of Mathematics, Vol. 33, Springer-Verlag, Berlin (1997).

5. P. Embrechts and N. Veraverbeke, "Estimates for the probability of ruin with special emphasis on the possibility of large claims," Insur. Math. Econ., 1, No.1, 55-72 (1982).

6. C. M. Goldie and C. Klüppelberg, "Subexponential distributions," in: A Practical Guide to Heavy Tails, Birkhäuser, Boston, (1998), pp. 435-459.

7. R. M. Loynes, "The stability of a queue with non-independent interarrival and service times," Proc. Cambridge Philos. Soc., 58, 497-520 (1962).

8. A. M. Parulekar, "M/G/ $\infty$ input proceses, a versatile class of models for network traffic," Technical Research Report, T. R., 96-59 (2001).

9. L. Massoulie and A. Simonian. "Large buffer asymptotics for the queue with fractional Brownian input," $J$. Appl. Probab., 36, No. 3, 894-906 (1999).

10. A. B. M.Crovella, "Self-similar in world wide web traffic:evidence and possible causes," Perf. Eval. Rev., No. 24, 160-169 (1996).

11. I. Norros, "A storage model with self-similar input," Queueing Syst. Theory Appl., 16, No. 3-4, 387-396 (1994).

12. M. Parulekar, "Buffer engineering for $M / G / \infty$ input processes," Ph.D. Thesis, Graduate School of the University of Maryland, College Park, (2000).

13. M. Parulekar and A. Makowski, "Tail probabilities for a multiplexer with self- similar traffic," in Proceedings of INFOCOM, San Fransisco, USA (1996), pp. 1452-1459.

14. M. Parulekar and A. M. Makowski. "Tail probabilities for $M / G / \infty$ input processes. I. Preliminary asymptotics," Queueing Syst. Theory Appl., 27, No. 3-4, 271-296 (1997).

15. Paxson and S. Floyd, "Wide area traffic: The failure of poisson modeling," IEEE/ACM Trans. Netw., 3, 226-244 (1993).

16. S. Resnick and G. Samorodnitsky, "Steady-state distribution of the buffer content for $M / G / \infty$ input fluid queues," Bernoulli, 7, No. 2, 191-210 (2001).

17. N. Veraverbeke, "Asymptotic behaviour of Wiener-Hopf factors of a random walk," Stoch. Proc. Appl., 5, No. 1, 27-37 (1977).

18. A. Villani, Exact asymptotics of the overflow probability for a $D / G / 1 / \infty$ queue with LRD service times, Ph.D. Thesis, University of L'Aquila (Italy), Mathematics Department (2005). 